\title{
Characteristics of cysteine-requiring strains of Klebsiella isolated from urinary tract infections
}

\author{
C. J. McIVER and J. W. TAPSALL
}

Department of Microbiology. The Prince of Wales Hospital, Randwick, Sydney, New South Wales, Australia 2031

\begin{abstract}
Summary. Clinical and bacteriological findings in seven cases of urinary tract infection with cysteine-requiring strains of Klebsiella are described. The organisms were isolated from patients with long-standing urinary tract abnormalities and grew as small $(c .1 \mathrm{~mm})$ colonies on MacConkey agar. The organisms failed to grow in a minimal medium supplemented with sodium sulphate but grew when the medium was supplemented with cysteine sulphinic acid, sodium sulphide or L-cysteine. The smallest amount of cysteine required for optimal growth in a chemically defined medium was $20 \mathrm{mg} / \mathrm{L}$. Cysteine-requiring strains of Escherichia coli had previously been shown to require a similar amount of cysteine and to be unable to reduce sulphate to sulphite; this suggests a common influence in the selection of cysteine auxotrophs in vivo. However, the amino acid inhibited the growth of $E$. coli at concentrations which only slightly altered growth of the Klebsiella strains. Problems with the isolation, identification and sensitivity testing of cysteine-requiring Klebsiella were also observed and methods by which these may be minimised are suggested.
\end{abstract}

\section{Introduction}

Enterobacteriaceae with a variety of metabolic defects are sometimes isolated from patients with urinary tract infections. These strains are most often seen in the elderly who have chronic diseases, in patients with structural abnormalities of the urinary tract (Gillespie, 1952; Borderon and Horodniceanu, 1978) or in those who have received co-trimoxazole therapy (Tapsall et al., 1974).

Gillespie (1952) isolated and characterised strains with defects in sulphur metabolism that could be corrected, at least partially, by the provision of exogeneous cysteine and other sulphurcontaining compounds. Most of these urinary isolates were Escherichia coli and similar organisms have been recovered subsequently from blood cultures and wound abscesses (Tapsall and McIver, 1986). Gillespie (1952) also described one strain of "Bact. aerogenes" in his study of cysteine-requiring urinary isolates.

Although cystine-supplemented media are available to facilitate the isolation of these organisms (Mackey and Sandys, 1966; Bevis, 1968), a number of problems still exist with regard to the isolation, identification and susceptibility testing of cysteine-

Received 2 Nov. 1987; accepted 22 Dec. 1987. requiring $E$. coli. We report seven cases of urinary tract infection caused by cysteine-requiring klebsiellae and discuss difficulties peculiar to their isolation and characterisation. Experiments designed to define further the metabolic defects, and the requirement for cysteine of these strains, are also described. Additionally, the toxic effects of higher concentrations of this amino acid were examined and the results compared with those previously obtained with auxotrophic $E$. coli under similar conditions (McIver and Tapsall, 1987).

\section{Materials and methods}

\section{Bacteriological methods}

The organisms were isolated from urine on MacConkey agar (Oxoid) and growth quantified by the filter paper method of Leigh and Williams (1964). The requirement for cysteine was shown on Sensitest Agar (Oxoid) by demonstrating growth only around a paper disk saturated with $0.63 \mathrm{mM}$ L-cysteine hydrochloride (Sigma) (Tapsall and McIver, 1986). The isolates were identified by the methods of Cowan (1974) with conventional bacteriological identification media (Oxoid or Difco) and by the Analytical Profile Index 20E (API systems, La Balme les Grottes, France). Antibiotic susceptibility tests were performed by a modification of the calibrated dichotomous sensitivity (CDS) method (Bell, 1975) on Sensitest 
Agar. The inocula were prepared in physiological saline (sensitivity and conventional identification tests) or sterile water (API system) with and without a supplement of Lcysteine $\mathrm{HCl} 100 \mathrm{mg} / \mathrm{L}(0.63 \mathrm{~mm})$.

The phenotypes of the isolates were assigned according to their response to intermediates of the sulphate assimilatory pathway (Jones-Mortimer, 1968) by methods previously described for cysteine-requiring $E$. coli (Tapsall and McIver, 1986). Briefly, a suspension of the organism was prepared from an overnight culture in minimal medium (Pasternak, 1962) containing 0.63 mM L-cysteine $\mathrm{HCl}$. After washing, this suspension was adjusted to $10^{4} \mathrm{cfu} / \mathrm{ml}$ as determined by surface viable counts (Miles et al., 1938). A volume of $0.025 \mathrm{ml}$ of this suspension was then inoculated by means of a Steers-type replicating device on to minimal agar medium with and without sodium sulphate, cysteine sulphinic acid, sodium sulphide or L-cysteine $\mathrm{HCl}$ in concentrations previously described (Tapsall and McIver, 1986). The inoculated media were incubated aerobically for up to 3 days at $37^{\circ} \mathrm{C}$ and examined daily for growth.

The cysteine requirements of three auxotrophic strains were determined in a chemically defined medium (Davis and Mingioli, 1950) by methods previously described (McIver and Tapsall, 1987). Briefly, the organisms were grown overnight in defined broth supplemented with $0.63 \mathrm{mM} \mathrm{L}$-cysteine $\mathrm{HCl}$; the cultures were then centrifuged and washed three times in physiological saline and inoculated into $2.5 \mathrm{ml}$ of defined broth to give an initial optical density (OD) at $640 \mathrm{~nm}$ of 0.05 in a Spectronic 20 spectrophotometer (Milton Roy, Rochester, NY, USA). The inoculated broths were incubated at $37^{\circ} \mathrm{C}$ in a shaking waterbath. The specific growth rates, length of lag phase and growth yield were calculated from growth curves constructed from OD readings taken at intervals of $30 \mathrm{~min}$ until the cultures reached stationary phase (Nagy et al., 1969). The OD readings were related to surface viable counts (Miles et al., 1938). Solutions of cysteine were freshly prepared, filtered and added to the medium immediately before each experiment and the $\mathrm{pH}$ of all media was adjusted to 7.0 before use. All experiments were performed in duplicate and repeated twice. The prototrophic strain Klebsiella pneumoniae ATCC 12657 was used as a control.

\section{Results}

The clinical features of the seven patients with urinary tract infections caused by cysteine-requiring isolates of Klebsiella are shown in table I.

The seven isolates grew poorly on MacConkey agar, producing small mucoid colonies $c .1 .0 \mathrm{~mm}$ in diameter. On medium supplemented with $0.63 \mathrm{~mm}$ cysteine, the colony size of the auxotrophs increased to about $3.0 \mathrm{~mm}$ in diameter and the typical morphological features of prototrophic strains of the genus were clearly recognisable. All strains were shown to be auxotrophic for cysteine by the paper disk method.

The organisms could not be identified by conventional techniques in the absence of cysteine supplementation. All seven strains failed to grow optimally in media used to test for lysine decarboxylase (Difco), for urease activity and for citrate utilisation (Oxoid). With the API 20E identification system, the strains failed to show a requirement for citrate and the Voges-Proskauer reactions were negative. Additionally, $p \mathrm{H}$ changes were slow to appear in the carbohydrate utilisation tests. Although some isolates were identified as "Klebsiella spp." in this

Table I. Clinical details of seven patients with urinary tract infections caused by cysteine-requiring Klebsiella

\begin{tabular}{|c|c|c|c|c|}
\hline Patient & Age & $\operatorname{Sex}$ & Associated clinical features & Outcome \\
\hline 1 & 77 & $\mathbf{M}$ & $\begin{array}{l}\text { Bilateral hydronephrosis; prostatism; diabetes } \\
\text { mellitus }\end{array}$ & $\begin{array}{l}\text { Organism eliminated with clavulanate-poten- } \\
\text { tiated amoxycillin }\end{array}$ \\
\hline 2 & 69 & $\mathbf{F}$ & $\begin{array}{l}\text { Recurrent UTI; renal calculi; analgesic } \\
\text { nephropathy }\end{array}$ & Failed to settle on cephalexin therapy \\
\hline 3 & 83 & $\mathbf{F}$ & $\begin{array}{l}\text { Synovitis of knee; incontinence; residual urine } \\
\text { after voiding }\end{array}$ & Not recorded \\
\hline 4 & 65 & $\mathbf{F}$ & $\begin{array}{l}\text { Renal transplant } 14 \text { years; immunosuppressed; } \\
\text { diabetes mellitus }\end{array}$ & Responded to gentamicin therapy \\
\hline 5 & 28 & $\mathrm{~F}$ & $\begin{array}{l}\text { Severe reflux nephropathy with scarring; recur- } \\
\text { rent UTI }\end{array}$ & $\begin{array}{l}\text { Clinical pyelonephritis responded to co-trimox- } \\
\text { azole therapy }\end{array}$ \\
\hline 6 & 74 & $\mathbf{M}$ & $\begin{array}{l}\text { Vesico-colic fistula and carcinoma of sigmoid; } \\
\text { prostatism }\end{array}$ & $\begin{array}{l}\text { Infection resolved with closure of fistula and } \\
\text { transurethral resection of prostate }\end{array}$ \\
\hline 7 & 69 & $\mathrm{~F}$ & $\begin{array}{l}\text { Analgesic nephropathy; un-united fracture of } \\
\text { humerus }\end{array}$ & $\begin{array}{l}\text { Died of unrelated causes. Granular, contracted } \\
\text { kidneys at autopsy }\end{array}$ \\
\hline
\end{tabular}


system, four strains produced profile numbers which did not allow identification. Growth in both systems was enhanced when the inocula were prepared in solutions supplemented with $0.63 \mathrm{mM}$ L-cysteine $\mathrm{HCl}$. By this procedure, six of the seven auxotrophs were identified as $K$. aerogenes $(K$. pneumoniae - sensu lato) by the methods of Cowan (1974) and as $K$. pneumoniae by the API system. The other strain was identified by the methods of Cowan (1974) as K. ozaenae, and by the API profile number as an unspeciated member of the genus Klebsiella.

None of the seven strains grew on Sensitest Agar when the inoculum was prepared in saline but when the saline suspensions were supplemented with cysteine $\mathrm{HCl}$, the organisms grew well. The sensitivities of the organisms to six antimicrobial agents tested by the CDS method (Bell, 1975) are shown in table II. One strain was resistant to all six agents; additional tests showed it to be resistant also to tobramycin and kanamycin but sensitive to amikacin, netilmicin, cefotaxime and to the combination of amoxycillin and clavulanic acid.

All strains grew in minimal medium supplemented with cysteine sulphinic acid, sodium sulphide or cysteine, but not on unsupplemented medium or in the presence of sodium sulphate. The effects of increasing concentrations of cysteine on growth yield and specific growth rate are shown in the figure. The three auxotrophs tested each produced a similar growth curve in response to increasing levels of cysteine. The strains failed to grow in cysteine-deficient broth, but growth yields improved as the cysteine concentration was increased to $20 \mathrm{mg} / \mathrm{L}(0.13 \mathrm{~mm})$. Above this concentration, up to a level of $1.27 \mathrm{~mm}$ cysteine $(200 \mathrm{mg} / \mathrm{L})$, similar growth yields were observed for each strain.

There were noticeable differences between the auxotrophic strains and the prototrophic control with regard to the specific growth rate at cysteine concentrations in the range $20-300 \mathrm{mg} / \mathrm{L}(0.13-$ $1.90 \mathrm{~mm})$. With the auxotrophic strains there was a gradual increase in the specific growth rate as the concentration was increased, but there was no concomitant alteration in the growth yield. In contrast, the specific growth rate of the prototrophic control was similar at all concentrations of cysteine up to $300 \mathrm{mg} / \mathrm{L}$, also with no marked changes in the growth yield. The specific growth rate and growth yield of all strains decreased slightly at concentrations of cysteine above $400 \mathrm{mg} / \mathrm{L}$. The lag phase of all strains was slightly increased at cysteine concentrations in the range $20-300 \mathrm{mg} / \mathrm{L}$ but was reduced in the presence of higher levels of the amino acid.

\section{Discussion}

The only previous report of the isolation of cysteine-requiring Klebsiella was that of Gillespie (1952) who found a strain that "resembled Bact. aerogenes" in the urine of an elderly male patient. Only a limited amount of information on the strain was provided in this report, which dealt principally with cysteine-requiring $E$. coli. It was noted that auxotrophic $E$. coli were isolated most commonly from elderly patients with chronic renal tract problems. Six of the seven infections caused by cysteine-requiring klebsiellae which we report were also in elderly patients with chronic renal problems. One younger patient was aged 28 years at the time of her infection; she suffered from long-standing reflux nephropathy and renal scarring was reported on intravenous pyelography.

The seven strains described here exhibited six different sensitivity patterns when tested against a standard set of six urinary antimicrobial agents.

Table II. Antibiotic susceptibility of cysteine-requiring isolates of Klebsiella tested by the method of Bell (1975)

\begin{tabular}{|c|c|c|c|c|c|c|}
\hline \multirow[b]{2}{*}{ Organism* } & \multicolumn{6}{|c|}{ Susceptibility to } \\
\hline & Sulphonamide & Trimethoprim & Ampicillin & Nalidixic acid & Nitrofurantoin & Gentamicin \\
\hline$K$. aerogenes & $\mathbf{R}$ & $\mathbf{R}$ & $\mathbf{R}$ & $\mathbf{R}$ & $\mathbf{R}$ & $\mathbf{R}$ \\
\hline$K$. aerogenes & $\mathrm{R}$ & $\mathbf{R}$ & $\mathbf{R}$ & $\mathbf{R}$ & $\mathbf{R}$ & $\mathbf{S}$ \\
\hline$K$. aerogenes & $\mathrm{S}$ & $\mathrm{S}$ & $\mathrm{R}$ & $\mathbf{S}$ & $\mathbf{R}$ & $\mathbf{S}$ \\
\hline$K$. aerogenes & $\mathrm{R}$ & $\mathbf{R}$ & $\mathbf{R}$ & $\mathbf{R}$ & $\mathbf{R}$ & $\mathbf{S}$ \\
\hline$K$. aerogenes & $\mathrm{S}$ & $\mathbf{S}$ & $\mathbf{R}$ & $\mathbf{S}$ & $\mathrm{S}$ & $\mathbf{S}$ \\
\hline K. aerogenes & $\mathrm{S}$ & $\mathbf{R}$ & $\mathrm{R}$ & $\mathrm{S}$ & $\mathrm{S}$ & $\mathbf{S}$ \\
\hline K. ozaenae & $\mathrm{R}$ & $\mathrm{S}$ & $\mathbf{R}$ & $\mathbf{S}$ & $\mathrm{S}$ & $\mathrm{S}$ \\
\hline
\end{tabular}

$R=$ resistant $; \mathbf{S}=$ sensitive .

* Identification of isolates according to Cowan (1974). 

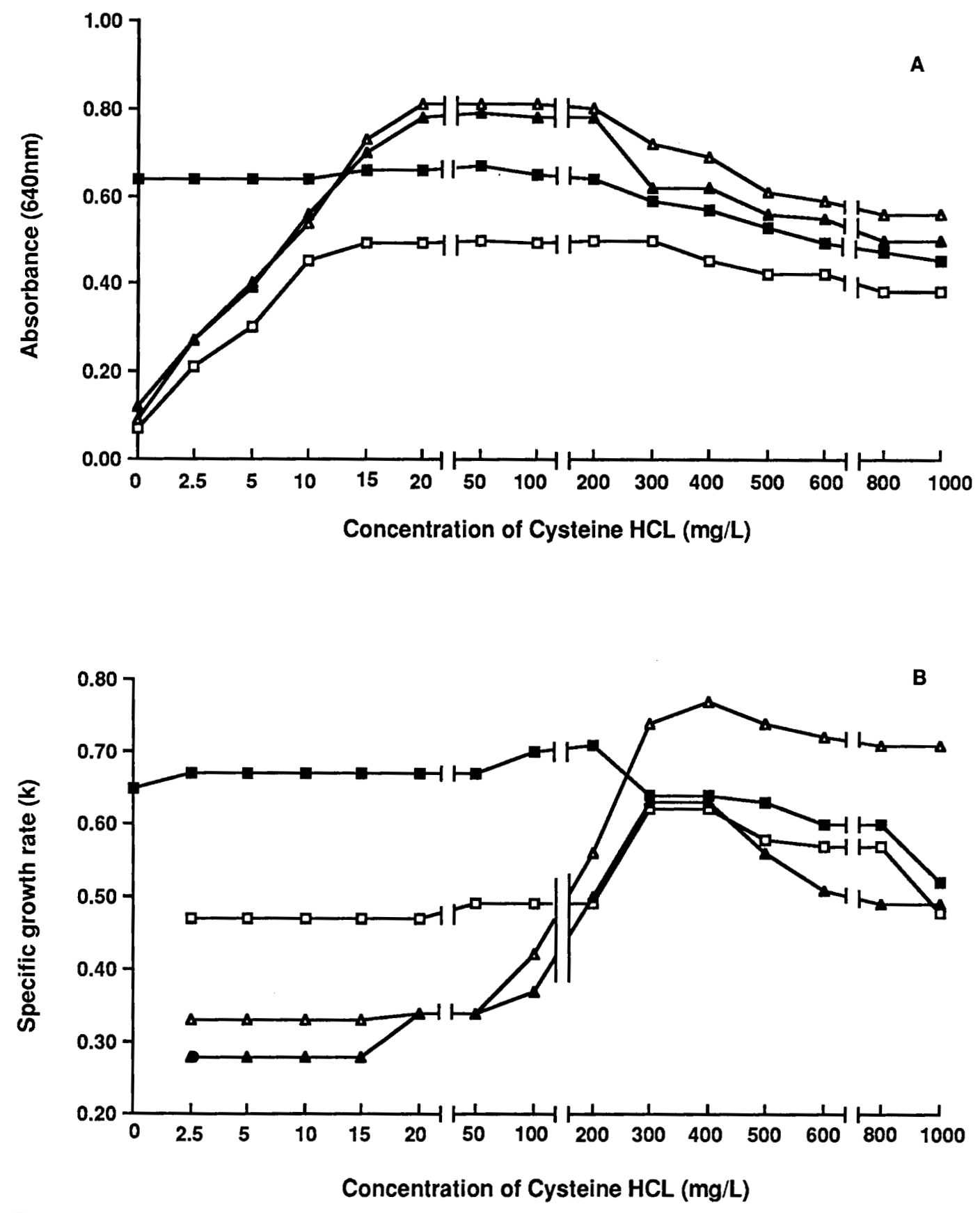

Figure. Growth yield (A) and specific growth rate (B) of three cysteine-requiring klebsiellae $(\square, \Delta, \Delta)$ and prototrophic control K. pneumoniae ATCC 12657 ( $)$ in Davis and Mingoli medium supplemented with increasing concentrations of L-cysteine.

Several of the auxotrophs appeared to be more resistant to antibiotics than other klebsiellae isolated from the urinary tract over the same period and this may reflect repeated exposure to antimicrobial agents in chronically infected patients. A number of problems arise in testing the sensitivity of auxotrophic strains in vitro. Not all media will fully support the growth of these strains and the presence of cysteine may affect the action of some antibiotics. Nonetheless antibiotic therapy based on the results of the in-vitro sensitivity tests appeared to be successful in most cases described 
here. One exception was patient 5 who responded clinically and bacteriologically to co-trimoxazole therapy, although the organism appeared to be resistant to this combination in vitro.

Cysteine-requiring strains represented about $1.2 \%$ of all isolates of Klebsiella over the period in which they were identified. This figure is similar to that given for the isolation of cysteine-requiring $E$. coli $(1.5 \%$ of isolates of this species) in the study of Borderon and Horodniceanu (1978) and indicates that factors responsible for the selection of cysteine auxotrophs in the urinary tract are not restricted to a single genus. This point is further emphasised by the finding that the defect in sulphur metabolism in the Klebsiella strains is similar to that defined previously in naturally occurring cysteine-requiring $E$. coli (McIver and Tapsall, 1987) in that the auxotrophic $E$. coli and Klebsiella both lack the ability to reduce sulphate to sulphite. This defect imposes a requirement for an exogenous source of suitable sulphur-containing compounds; cysteine and its oxidation product cystine, if present, provide "end product rescue" for the auxotrophs. These compounds have been shown to be present in the urine of fasting patients at concentrations of 2$4 \mathrm{mg} / \mathrm{L}(16.5-33.0 \mu \mathrm{M})$ and $10-15 \mathrm{mg} / \mathrm{L}(41.6-$ $62.4 \mu \mathrm{M}$ ) respectively (Brigham et al., 1960).

The relative lack of reports of cysteine-requiring klebsiellae in comparison to auxotrophic $E$. coli may arise from the much higher rate of isolation of $E$. coli from the urinary tract. Moreover, these

\section{REFERENCES}

Bell S M 1975 The CDS disc method of antibiotic sensitivity testing (calibrated dichotomous sensitivity test). Pathology 7 Suppl: $1-48$.

Bevis T D 1968 A modified electrolyte deficient culture medium. Journal of Medical Laboratory Technology 25:38-41.

Borderon E, Horodniceanu T 1978 Metabolically deficient dwarf-colony mutants of Escherichia coli: deficiency and resistance to antibiotics of strains isolated from urine culture. Journal of Clinical Microbiology 8:629-634.

Brigham M P, Stein W H, Moore S 1960 The concentrations of cysteine and cystine in human blood plasma. Journal of Clinical Investigation 39 : 1633-1638.

Cowan S T 1974 Cowan and Steel's Manual for the identification of medical bacteria, 2nd ed. Cambridge University Press, Cambridge, pp 103-110.

Davis B D, Mingioli E S 1950 Mutants of Escherichia coli requiring methionine or vitamin $\mathrm{B}_{12}$. Journal of Bacteriology 60: $17-28$.

Gillespie W A 1952 Biochemical mutants of coliform bacilli in infections of the urinary tract. Journal of Pathology and Bacteriology 64:551-557.

Jones-Mortimer M C 1968 Positive control of sulphate reduction in Escherichia coli. Isolation, characterization and mapping strains may be often misidentified, because failure of some routinely used media to support growth fully will cause difficulties in isolation, identification and sensitivity testing. These problems are partly corrected when exogenous cysteine is available, although Gillespie (1952) noted that additional unidentified factors aid the growth of cysteinerequiring $E$. coli.

We demonstrated that the auxotrophs and the control strain yielded similar growth in the presence of cysteine concentrations of $\geqslant 20 \mathrm{mg} / \mathrm{L}$. However, the specific growth rate was lower for auxotrophic strains and only approached that of the prototrophic control in the presence of cysteine at the relatively high concentration of $300 \mathrm{mg} / \mathrm{L}$. When the specific growth rates of prototrophic and auxotrophic $E$. coli were compared in earlier experiments (McIver and Tapsall, 1987), no such differences were observed. A further difference between the two genera was observed when toxic effects of higher concentrations of cysteine on $E$. coli and Klebsiella were compared. Toxic effects on $E$. coli occurred when the amino acid was present in concentrations of $\geqslant 250 \mathrm{mg} / \mathrm{L}(1.59 \mathrm{~mm}$ ) (McIver and Tapsall, 1987). In contrast, only slight reductions in growth yield and growth rate were observed with either prototrophic or auxotrophic klebsiellae in concentrations of cysteine up to $1000 \mathrm{mg} / \mathrm{L}$.

We thank Mr M. Wilson of The University of New South Wales for the gift of Klebsiella pneumoniae ATCC 12657. of cysteine-less mutants of E. coli K12. Biochemical Journal 110:589-595.

Leigh D A, Williams J D 1964 Method for the detection of significant bacteriuria in large groups of patients. Journal of Clinical Pathology 17: 498-503.

Mackey J P, Sandys G H 1966 Diagnosis of urinary infections. British Medical Journal $1: 1173$.

McIver C J, Tapsall J W 1987 Cysteine requirements of naturally occurring cysteine auxotrophs of Escherichia coli. Pathology 19:361-363.

Miles A A, Misra S S, Irwin E D 1938 The estimation of the bactericidal power of the blood. Journal of Hygiene 38: 732749.

Nagy Z, Kari C, Hernádi F 1969 Growth of Escherichia coli cells in the presence of cysteine on sulphate-deficient media. Archiv für Mikrobiologie 65:391-400.

Pasternak C A 1962 Sulphate activation and its control in Escherichia coli and Bacillus subtilis. Biochemical Journal 85: 44-49.

Tapsall J W, McIver C J 1986 Septicaemia caused by cysteinerequiring isolates of Escherichia coli. Journal of Medical Microbiology 22:379-382.

Tapsall J W, Wilson E, Harper J 1974 Thymine dependent strains of Escherichia coli selected by trimethoprim-sulphamethoxazole therapy. Pathology 6:161-167. 